\title{
Far-Infrared Irradiation Inhibits Adipogenic Differentiation and Stimulates Osteogenic Differentiation of Human Tonsil-Derived Mesenchymal Stem Cells: Role of Protein Phosphatase 2B
}

\author{
Ha Yeong Kim ${ }^{a, b}$ Yeonsil Yu $u^{a, c}$ Se-Young Oh ${ }^{a} \quad$ Kang-Kyun Wang ${ }^{d}$ \\ Yong-Rok Kim ${ }^{d}$ Sung-Chul Junge Han Su Kim ${ }^{b}$ Inho Jo ${ }^{a}$
}

\begin{abstract}
aDepartment of Molecular Medicine, College of Medicine, Ewha Womans University, Seoul, Republic of Korea, 'bepartment of Otorhinolaryngology-Head and Neck Surgery, College of Medicine, Ewha Womans University, Seoul, Republic of Korea, 'Stem Cell and Regenerative Bioengineering Institute, Kangstem Biotech Co., Ltd., Biotechnology Center, Seoul National University, Seoul, Republic of Korea, dDepartment of Chemistry, College of Science, Yonsei University, Seoul, Republic of Korea, eDepartment of Biochemistry, College of Medicine, Ewha Womans University, Seoul, Republic of Korea
\end{abstract}

\section{Key Words}

Far-infrared irradiation • Tonsil-derived mesenchymal stem cells • Adipogenesis • Osteogenesis - Protein phosphatase 2B

\begin{abstract}
Background/Aims: Far-infrared (FIR) irradiation has been reported to exhibit various biological effects including improvement of cardiovascular function. However, its effect on the differentiation of stem cells has not been studied. Using tonsil-derived mesenchymal stem cells (TMSC), we examined whether and how FIR irradiation affects adipogenic or osteogenic differentiation. Methods: TMSC were exposed to FIR irradiation (3-25 $\mu \mathrm{m}$ wavelength) for various times $(0,30$, or $60 \mathrm{~min})$, and then adipogenic or osteogenic differentiation was induced for 14 days with its respective commercially available differentiation medium. At the end of the differentiation, the cells were stained using Oil red $\mathrm{O}$ or Alizarin red $\mathrm{S}$ solution, and the expression of differentiation-specific proteins was analyzed by western blotting. Results: FIR irradiation did not alter cell viability or the expression of MSC-specific surface antigens (CD14, CD34, CD45, CD73, CD90, and CD105) in TMSC. However, FIR irradiation significantly inhibited adipogenic differentiation of TMSC, as evidenced by decreased Oil red O staining as well as protein expression of peroxisome proliferator-activated receptor $\gamma$ and fatty acid binding protein 4. In contrast, FIR irradiation induced osteogenic differentiation, as evidenced by increased Alizarin red $\mathrm{S}$ staining as well as protein expression of osteocalcin and alkaline
\end{abstract}




\section{Cellular Physiology Cell Physiol Biochem 2019;52:240-253

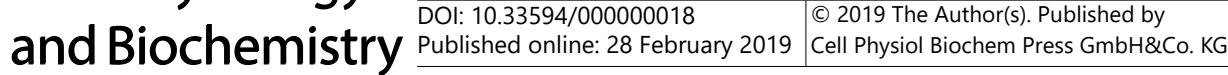 \\ Kim et al.: Far-Infrared Irradiation and Mesenchymal Stem Cell Differentiation}

phosphatase. Treatment with heat alone did not inhibit the adipogenic differentiation of TMSC, suggesting that the inhibitory effect on adipogenic differentiation was not due to heat induced by FIR irradiation. However, heat alone did stimulate osteogenic differentiation, but to a lesser extent than FIR irradiation. Furthermore, FIR irradiation increased intracellular $\mathrm{Ca}^{2+}$ levels and the activity of protein phosphatase 2B (PP2B) in TMSC. Treatment with cyclosporin $A$, a specific PP2B inhibitor, reversed the inhibitory effect of FIR irradiation on adipogenic differentiation of TMSC, but had no effect on osteogenic differentiation. Conclusion: Our data demonstrate that FIR irradiation inhibits adipogenic differentiation but enhances osteogenic differentiation of TMSC; the inhibitory effect on adipogenic differentiation is non-thermal and mediated at least in part by activation of $\mathrm{Ca}^{2+}$-dependent PP2B.

(C) 2019 The Author(s). Published by

Cell Physiol Biochem Press GmbH\&Co. KG

\section{Introduction}

Stem cells are generally defined as undifferentiated cells capable of self-renewal and differentiation into specialized cells, and can be classified based on their origin [1]. Stem cells are largely classified into two groups; embryonic stem cells derived from totipotent cells of the early mammalian embryo [2] or mesenchymal stem cells (MSC) derived from a variety of adult tissues, including bone marrow, adipose, and umbilical cord tissue [3-6]. MSC can mainly be differentiated into skeletal cells, such as osteocytes, chondrocytes, and adipocytes $[7,8]$. We previously established tonsil-derived MSC (TMSC) from human palatine tonsils, and they have been showing great differentiation potentials into several cell types, including adipocytes, osteocytes, chondrocytes, Schwann cells, muscle cells, insulin-releasing cells, tenocytes, and parathyroid hormone-releasing cells [9-15]. In addition to their superior differentiation potential, TMSC are obtained by completely non-invasive tonsillectomy and proliferate faster ( $\sim 1.5$ times faster) than bone marrow-derived MSC (BM-MSC). Because of these advantages, TMSC have been proposed as a potential new MSC for clinical studies [16-18].

Far-infrared (FIR) is one of the three infrared regions, and is defined by the International Commission on Illumination as an invisible electromagnetic wave with a wavelength of 3-1000 $\mu \mathrm{m}$ [19]. FIR irradiation is known to contribute to a variety of biological effects including improved vascular function [20], which is attributable in part to upregulation of the vasodilator nitric oxide (NO) in endothelial cells (EC) [21]. Furthermore, FIR irradiation has also been reported to inhibit cancer cell proliferation through regulation of heat shock protein 70A [22]. Although a single recent paper described the effect of FIR irradiation on the basic properties of murine BM-MSC, such as proliferation and survival [23], the effect of FIR on differentiation has not been reported.

Protein phosphatase 2B (PP2B, also known as calcineurin), a $\mathrm{Ca}^{2+} /$ calmodulindependent serine/threonine phosphatase, is an important mediator of intracellular $\mathrm{Ca}^{2+}$ signaling in a number of cell types, and is inhibited by cyclosporin A (CsA) and FK506 [24, 25]. A previous study revealed that increased activity of $\mathrm{Ca}^{2+}$-dependent PP2B in 3T3-L1 preadipocytes inhibits adipocyte differentiation by inhibiting the expression of peroxisome proliferator-activator receptor $\gamma(\mathrm{PPAR} \gamma)$ and CCAAT/enhancer-binding protein alpha (C/ $\mathrm{EBP} \alpha$ ] [26]. Furthermore, PP2B is expressed in osteoblasts and regulates bone formation in vitro and in vivo [27]. However, little is known about the direct effect of PP2B on adipogenic or osteogenic differentiation in stem cells.

We previously reported that FIR irradiation increases NO production through intracellular $\mathrm{Ca}^{2+}$ mobilization in bovine aortic EC (BAEC) [21]. Based on these findings, we investigated whether FIR irradiation also increases intracellular $\mathrm{Ca}^{2+}$ levels in TMSC and its potential effects on $\mathrm{Ca}^{2+}$-dependent PP2B activity, which in turn affects the differentiation potential of TMSC into adipocytes and osteocytes. 


\section{Cellular Physiology Cell Physiol Biochem 2019;52:240-253 \\ \begin{tabular}{l|l|l}
\hline DOI: 10.33594/000000018 & (2019 The Author(s). Published by
\end{tabular} \\ \begin{tabular}{l|l} 
Published online: 28 February 2019 & Cell Physiol Biochem Press GmbH\&Co. KG \\
\hline
\end{tabular} \\ Kim et al.: Far-Infrared Irradiation and Mesenchymal Stem Cell Differentiation}

\section{Materials and Methods}

\section{Isolation and culture of TMSC}

TMSC were isolated as described previously [9]. Briefly, TMSC were isolated from the tonsillar tissues of patients (aged $\leq 10$ years) undergoing tonsillectomy. Informed written consent was obtained from the legal guardians of the patients who participated in this study and the study protocol was approved by the Institutional Review Board (ECT-11-58-37) of Ewha Womans University Mokdong Hospital.

Isolated tonsil tissues were digested in RPMI-1640 medium (Invitrogen, Carlsbad, CA, USA) containing $210 \mathrm{U} / \mathrm{mL}$ collagenase type I (Invitrogen) and $10 \mathrm{mg} / \mathrm{mL}$ DNase (Sigma-Aldrich, St. Louis, MO, USA) and incubated for $30 \mathrm{~min}$ at $37^{\circ} \mathrm{C}$. After incubation, the digested tissues were filtered using a wire mesh and washed twice in Dulbecco's Modified Eagle Medium containing high glucose (4,500 mg/L) (DMEM-HG; Welgene Inc., Gyeongsan, Korea) and 20\% fetal bovine serum (FBS; Invitrogen) and once in DMEM-HG with $10 \%$ FBS. Mononuclear cells were obtained by density gradient centrifugation using Ficoll-Paque (GE Healthcare, Little Chalfont, UK) and plated in a T-150 culture flask (Corning, Tewksbury, MA, USA) in DMEMHG with 10\% FBS. After incubation for $48 \mathrm{~h}$, non-adherent cells were removed by washing and adherent cells were further incubated in the cell culture incubator at $37^{\circ} \mathrm{C}$ under $5 \% \mathrm{CO}_{2}$.

\section{FIR irradiation}

FIR irradiation was performed using an AC-driven constant-power lamp controller (Model No. S-O.T.H 9H, Saeik Medical Co., Ltd., Bucheon, Korea) with a ceramic infrared radiator (Model No. IOT/90-250, Elstein-Werk M. Steinmetz GmbH \& Co. KG, Northeim, Germany), as described previously [28] with slight modifications. The emission wavelength of the FIR radiator ranged from 3 to $25 \mu \mathrm{m}$, with a peak at $7.5 \mu \mathrm{m}$. The FIR radiator was pre-warmed for $30 \mathrm{~min}$ at room temperature, and then TMSC, cultured in a $60-\mathrm{mm}$ cell culture plate with $4 \mathrm{~mL}$ medium, were placed at a distance of $30 \mathrm{~cm}$ from the radiator and exposed for the indicated times $(0,30$, or $60 \mathrm{~min})$. TMSC were further incubated in the culture chamber at $37^{\circ} \mathrm{C}$ under $5 \% \mathrm{CO}_{2}$. In a separate experiment, TMSC were incubated at $38^{\circ} \mathrm{C}$ on a Chamlide heat incubator (Model No. CU-109, Live Cell Instrument, Seoul, Korea) for 30 min.

\section{Cell viability and proliferation}

Cell viability was assessed using 3-(4, 5-dimethylthiazol-2-yl)-2, 5-diphenyltetrazolium bromide (MTT) solution. Cells were seeded onto 48-well plates and exposed to FIR irradiation for the indicated times. MTT solution was added to each well, and the culture plate was incubated for $2 \mathrm{~h}$ in a $37^{\circ} \mathrm{C}$ incubator. The medium was removed and the purple formazan product was dissolved using dimethyl sulfoxide. Dissolved solutions were transferred to a 96-well plate and the absorbance was measured at $540 \mathrm{~nm}$ with a microplate reader. For the measurement of proliferation, TMSC exposed to FIR irradiation were further incubated for 18,24 , or $48 \mathrm{~h}$ and subjected to MTT assay. Proliferation was expressed as the absorbance at indicated times relative to that at time 0 .

\section{Fluorescence-activated cell sorting (FACS) analysis}

After exposure of TMSC to FIR irradiation for 0 or $30 \mathrm{~min}$, cells were collected and stained with phycoerythrin (PE)-conjugated anti-human CD14, CD34, CD73, and fluorescein isothiocyanate (FITC)conjugated anti-human CD45, CD90, CD105 antibodies. Stained cells were analyzed using a FACSCalibur system (BD Biosciences, San Diego, CA, USA). All antibodies were purchased from BD Biosciences.

\section{Adipogenic or osteogenic differentiation and drug treatments}

TMSC at a confluence of $80-90 \%$ were exposed to FIR irradiation for the indicated times and incubated in commercially available adipogenic or osteogenic differentiation medium (Cat. No. A10070-01 or A1007201; Invitrogen) for up to 14 days. The culture medium was changed every 3 or 4 days. In some experiments, TMSC were pretreated with CsA (0, 0.25, 0.5, and $1 \mu \mathrm{M}$; Sigma-Aldrich) before exposure to FIR irradiation and then subjected to differentiation. After differentiation, cells were fixed with $4 \%$ formalin for $30 \mathrm{~min}$ and washed with phosphate-buffered saline. Adipogenic differentiated cells were stained with Oil Red 0 solution and osteogenic differentiated cells with Alizarin red S solution for $1 \mathrm{~h}$ at room temperature. The remaining excessive staining solution was removed, and stained cells were visualized under a microscope. 


\section{Cellular Physiology Cell Physiol Biochem 2019;52:240-253

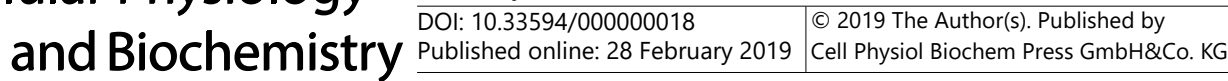 \\ Kim et al.: Far-Infrared Irradiation and Mesenchymal Stem Cell Differentiation}

\section{Western blot analysis}

Cells were extracted using lysis buffer containing Protease Inhibitor Cocktail ${ }^{\mathrm{TM}}$ (Roche Molecular Biochemicals, Indianapolis, IN, USA). The protein samples (equal quantities of $20 \mu \mathrm{g}$ ) were separated using SDS gel electrophoresis and transferred onto nitrocellulose membranes. The blots were probed with primary antibodies against transcriptional coactivator with PDZ-binding motif (TAZ), PPAR $\gamma$, or fatty acid binding protein 4 (FABP4) (each at a 1:1000 dilution; Cell Signaling Technology, Boston, MA, USA); osteocalcin (1:500; Santa Cruz Biotechnology, Santa Cruz, CA, USA); alkaline phosphatase (ALP; 1:1000; Abcam, Cambridge, UK); and glyceraldehyde 3-phosphate dehydrogenase (GAPDH; 1:2500 dilution; AbFrontier, Seoul, Korea) followed by the corresponding secondary antibodies, and the proteins were detected by enhanced chemiluminescence detection methods (Amersham, Buckinghamshire, UK).

\section{Intracellular calcium measurement}

The membrane-permeable calcium indicator dye Fluo-4 AM (Invitrogen) was used to measure intracellular $\mathrm{Ca}^{2+}$ levels as described previously [21]. Fluorescence images were obtained using a confocal microscope (LSM5 Pascall, Carl ZEISS, Oberkochen, Germany).

\section{Protein phosphatase $2 B(P P 2 B)$ activity assay}

PP2B activity was measured using the PP2B cellular activity kit (Enzo Life Sciences, Farmingdale, NY, USA) with minor modification. Briefly, TMSC were exposed to FIR irradiation for 0 or 30 min and rinsed in ice-cold tris-buffered saline $(20 \mathrm{mM}$ Tris, $\mathrm{pH} 7.2,150 \mathrm{mM} \mathrm{NaCl})$. After washing, cells were lysed in lysis buffer with protease inhibitors and centrifuged at 16,000 g for $20 \mathrm{~min}$. To remove free phosphate, the supernatant extracts were exposed to desalting column resin. The extracted supernatant (500 $\mu \mathrm{g}) \mathrm{was}$ immunoprecipitated using anti-PP2B antibody (Cell Signaling Technology) and washed three times with lysis buffer. The immunoprecipitated samples were assessed using a PP2B cellular activity kit (Enzo Life Sciences) as described in the instruction manual. PP2B activity was quantified by measuring absorbance at $620 \mathrm{~nm}$ and normalized using the controls.

\section{Statistical analysis}

All results are presented as the means \pm standard deviations (S.D.). Statistical significance was determined using Student's t-test. A value of $\mathrm{p}<0.05$ was considered significant. All experiments were performed at least three times.

\section{Results}

FIR irradiation does not affect cell viability, expression of MSC-specific surface antigen markers, and proliferation of TMSC

To assess whether FIR irradiation affects the viability of TMSC, cells were exposed to FIR irradiation for 0,30 , or 60 min at room temperature. Results of the MTT assay showed that FIR irradiation did not affect cell viability, even at the maximum exposure time of $60 \mathrm{~min}$ (Fig. 1A). Next, we examined whether FIR irradiation alters the surface immunophenotypic character of TMSC. FACS analysis revealed that the surface markers tested in this study were not altered by FIR irradiation (Fig. 1B); TMSC in the absence or presence of FIR irradiation were negative for hematopoietic surface markers (CD14, CD34, and CD45) and displayed significant positive expression of MSC-specific surface markers (CD73, CD90, and CD105). Cell proliferation was also tested by further incubation of TMSC for $0,18,24$, or $48 \mathrm{~h}$ after FIR irradiation. FIR irradiation affected unlikely TMSC growth compared with untreated controls (Fig. 1C). Together, these results suggest that FIR irradiation did not alter the viability, surface antigen expression, or proliferation of TMSC under our experimental conditions. 


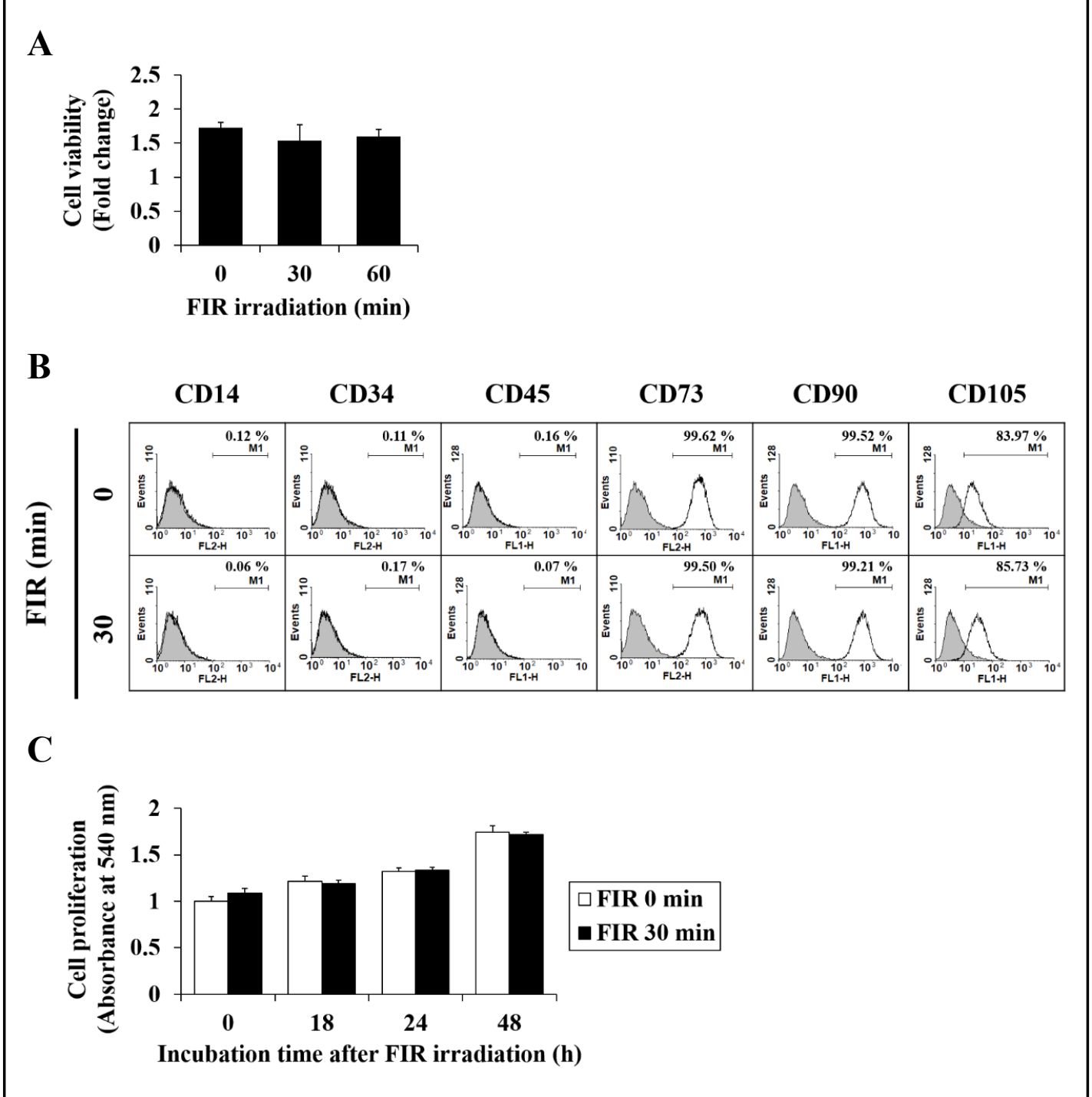

Fig. 1. FIR irradiation does not alter cell viability, expression of MSC-specific surface antigen markers, and proliferation of TMSC. TMSC were exposed to FIR irradiation at room temperature for 0 , 30, and 60 min. (A) The viability of TMSC was measured by MTT assay. (B) The profiles of MSC-specific surface markers were analyzed by flow cytometry. (C) After FIR irradiation (30 min), the cells were further incubated for 0,24 , and $48 \mathrm{~h}$ in the cell culture incubator and cell growth was determined by MTT assay. The values of cell growth are expressed as means \pm S.D $(n=3)$.

Acute exposure to FIR irradiation inhibits adipogenic differentiation but promotes osteogenic differentiation of TMSC

To investigate whether FIR irradiation affects the differentiation of TMSC, cells were exposed to FIR irradiation for 30 and $60 \mathrm{~min}$ and then further differentiated using commercially available adipogenic or osteogenic differentiation medium for up to 14 days. As shown in Fig. 2A, lipid accumulation was significantly suppressed by FIR irradiation in a time-dependent manner. The expression levels of the adipocyte-specific markers PPAR $\gamma$ and FABP4 were also significantly downregulated (Fig. 2B). In contrast, as exposure time of FIR irradiation increased, mineralization was promoted and the protein expression of osteocytespecific markers osteocalcin and ALP also significantly increased (Fig. 2C, D). 
A.

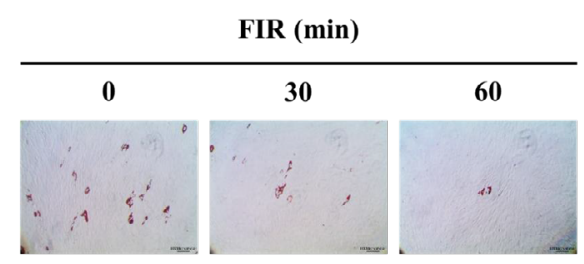

B.
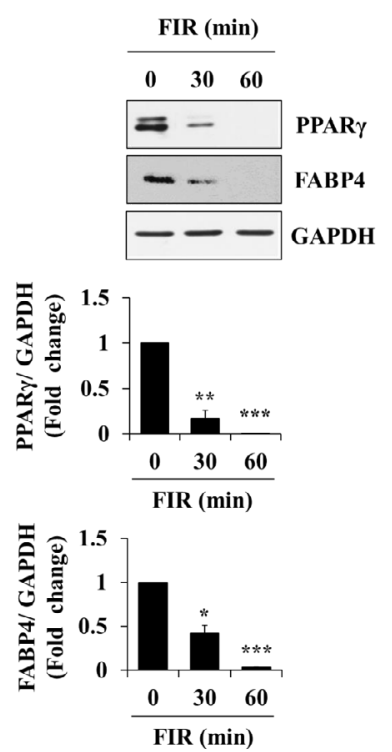

C.

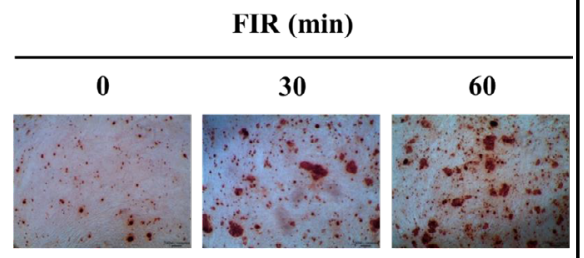

D.
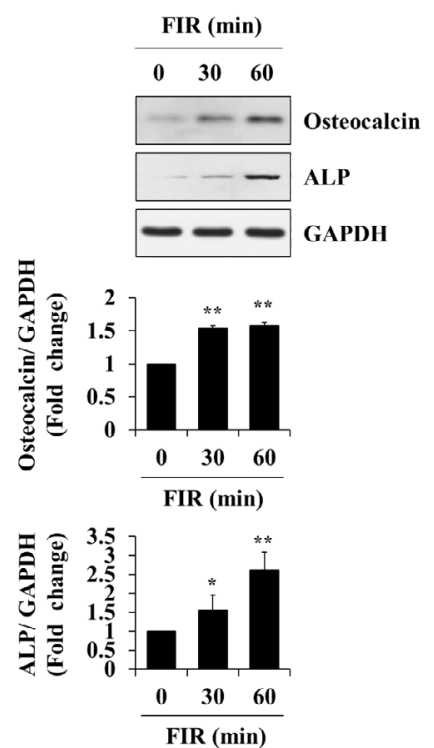

Fig. 2. Acute exposure to FIR irradiation inhibits adipogenic differentiation and promotes osteogenic differentiation of TMSC. TMSC were exposed to FIR irradiation for 0, 30, and $60 \mathrm{~min}$ and further incubated in commercially available adipogenic or osteogenic differentiation medium for up to 14 days. (A) The accumulation of lipid droplets was assessed using Oil red 0 staining. Scale bar $=100 \mu \mathrm{m}$. (B) Protein levels of PPAR $\gamma$ and FABP4 were measured by western blot analysis. GAPDH was used as a loading control for normalization. (C) Mineralization was assessed using Alizarin red S staining. Scale bar $=200 \mu \mathrm{m}$. (D) Protein levels of osteocalcin and ALP were measured by western blot analysis. GAPDH was used as a loading control for normalization. The results are representative of three independent experiments, and each bar represents the mean \pm S.D. Differences were statistically significant at ${ }^{*} \mathrm{p}<0.05,{ }^{* *} \mathrm{p}<0.01$, and ${ }^{* * *} \mathrm{p}<0.001$.

The inhibitory activity of FIR irradiation on adipogenic differentiation is not caused by thermal effects

Previously, we reported that FIR irradiation increases the temperature of the culture medium to $38 \pm 1^{\circ} \mathrm{C}[28]$. Based on this finding, we examined whether the heat alone induced by FIR irradiation regulates adipogenesis or osteogenesis of TMSC. Compared with untreated control cells, heat treatment $\left(\right.$ at $38^{\circ} \mathrm{C}$ ) alone using the Chamlide heat incubator did not inhibit adipogenic differentiation of TMSC as evidenced by Oil red 0 staining (Fig. 3A) and the expression of PPAR $\gamma$ and FABP4 (Fig. 3B). Next, we tested the thermal effect of FIR irradiation on osteogenesis of TMSC. Although heat alone did stimulate osteogenic differentiation of TMSC, as evidenced by Alizarin red S staining, its effect was smaller than that with FIR irradiation (Fig. 3C). Furthermore, heat alone also increased osteocalcin expression but to a lesser extent than FIR irradiation (Fig. 3D). However, the protein expression of ALP was not significantly different between heat-treated and FIR-irradiated TMSC. Together, these results suggest that the inhibitory effect of FIR irradiation on adipogenic differentiation of TMSC is unlikely to be due to the thermal effect caused by FIR irradiation. 


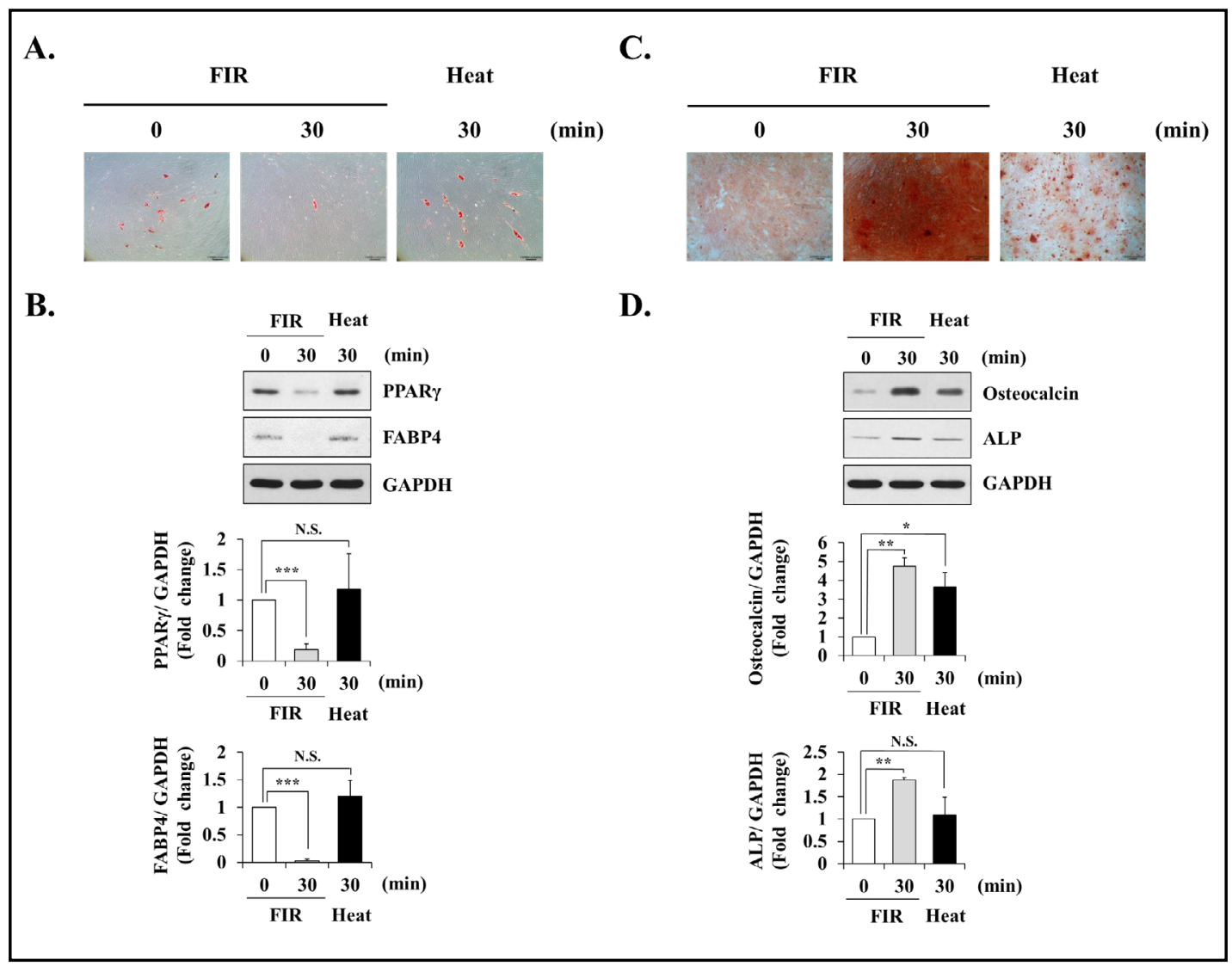

Fig. 3. Effects of thermal treatment on adipogenic or osteogenic differentiation of TMSC. TMSC were exposed to FIR irradiation or heat $\left(38^{\circ} \mathrm{C}\right)$ using the Chamlide heat incubator for $30 \mathrm{~min}$ and further incubated in differentiation medium for up to 14 days. (A) Accumulation of lipid droplets, (B) protein levels of PPAR $\gamma$, FABP4, and GAPDH, (C) mineralization, and (D) protein levels of osteocalcin, ALP, and GAPDH were measured as described in the legend of Fig. 2. The results are representative of three independent experiments, and each bar represents the mean \pm S.D. Differences were statistically significant at ${ }^{*} \mathrm{p}<0.05$, ${ }^{* *} \mathrm{p}<0.01$, and ${ }^{* * *} \mathrm{p}<0.001$. N.S., not significant.

Transcriptional coactivator with PDZ-binding motif (TAZ) is not involved in the adipogenic or osteogenic differentiation of TMSC under our experimental conditions

TAZ is a well-known transcriptional modulator that regulates adipogenesis and osteogenesis of MSC; it inhibits adipogenic differentiation and promotes osteogenic differentiation [29]. Based on this knowledge, we investigated whether TAZ regulates the FIR irradiation-induced decrease in adipogenesis and increase in osteogenesis of TMSC. If this is the case, we expected that FIR irradiation would increase TAZ protein expression. Under our conditions, however, FIR irradiation for $30 \mathrm{~min}$ decreased the protein level of TAZ (Fig. 4), suggesting no evidence for involvement of TAZ in the adipogenic or osteogenic differentiation of TMSC by FIR irradiation.

Cyclosporin A, a specific inhibitor of protein phosphatase $2 B(P P 2 B)$ activity, reverses the effect of FIR irradiation on adipogenic differentiation but not osteogenic differentiation

Several studies have shown that PP2B, known as an intracellular $\mathrm{Ca}^{2+}$-dependent phosphatase, exhibits a regulatory role in adipogenesis or osteogenesis [26, 27]. These reports, together with our previous study showing increased intracellular $\mathrm{Ca}^{2+}$ levels in FIR-irradiated BAEC [21], prompted us to examine whether PP2B is also involved in FIR irradiation-mediated effects on each type of differentiation in TMSC. As in BAEC, we found that FIR irradiation increased intracellular $\mathrm{Ca}^{2+}$ levels in TMSC (Fig. 5A). Furthermore, PP2B 
activity was significantly elevated in FIR-irradiated TMSC (Fig. 5B). To examine whether PP2B is also involved in the effect of FIR irradiation on the differentiation of TMSC, we used a specific inhibitor of PP2B, CsA. As shown in Fig. 6A, treatment with CsA (up to $0.5 \mu \mathrm{M}$ ) clearly reversed the decrease in lipid accumulation induced by FIR irradiation. This reversal also occurred at a higher dose of CsA $(1 \mu \mathrm{M})$, but without statistical significance. Similarly, the inhibitory effect of FIR irradiation on PPAR $\gamma$ and FABP4 expression was also significantly reversed when TMSC were treated with CsA at concentrations up to 0.5 $\mu \mathrm{M}$ (Fig. 6B) whereas no significant reversal occurred in TMSC treated with $1 \mu \mathrm{M}$ CsA. Interestingly, none of the tested doses of CsA altered FIR irradiation-stimulated osteogenic differentiation with respect to mineralization (Fig. 6C) and protein expression of osteocalcin and ALP (Fig. 6D). These data suggest an important role for PP2B in regulating adipogenesis, but not osteogenesis, of TMSC induced by FIR irradiation.

\section{Discussion}

Previous studies have shown that FIR irradiation has therapeutic effects in various disease models, including models of cardiovascular disease and cancer [30, 31]. For the last decade, several types of cells, including EC and cancer cells [21, 22], have

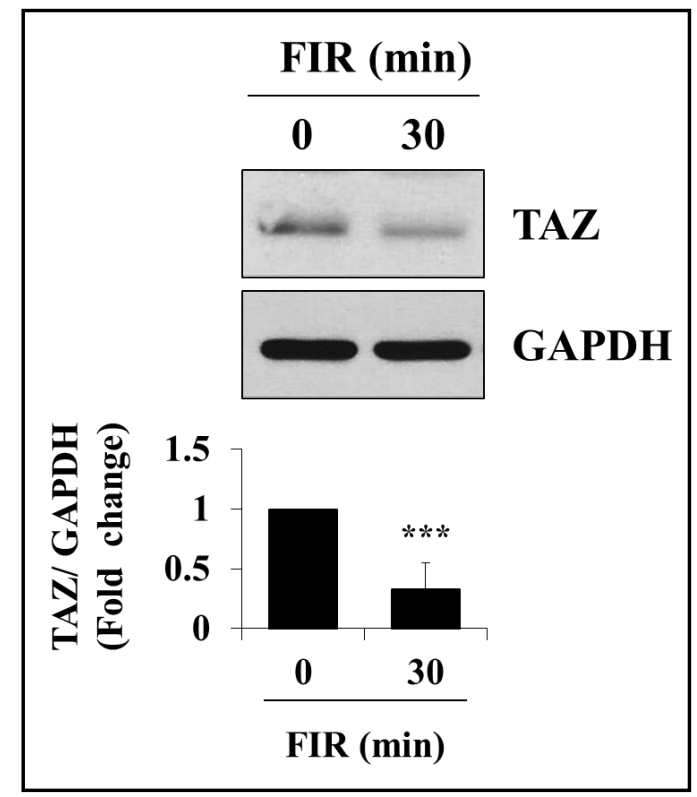

Fig. 4. Expression of $\mathrm{TAZ}$ as a modulator of MSC differentiation. TMSC were exposed to FIR irradiation for $30 \mathrm{~min}$. Protein levels of TAZ and GAPDH were measured as described in the legend of Fig. 2. The results are representative of three independent experiments, and the bar represents the mean \pm S.D. Difference was statistically significant at $* * * p<0.001$.

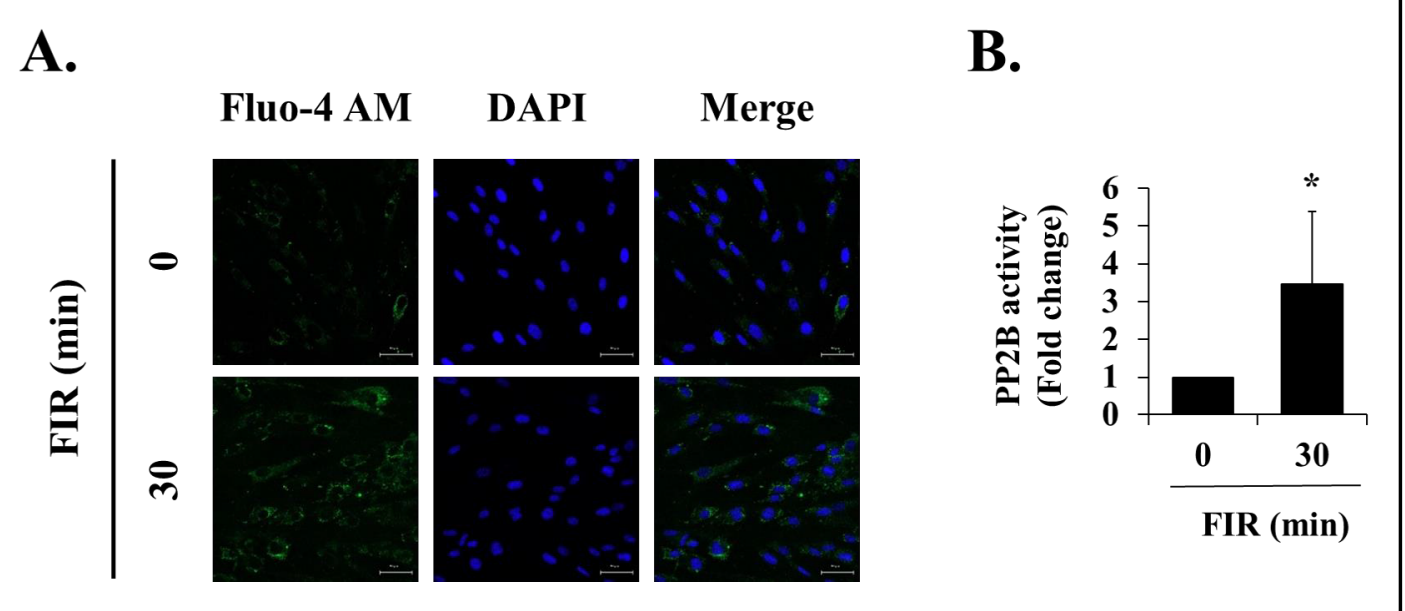

Fig. 5. FIR irradiation increases the activity of $\mathrm{Ca}^{2+}$-mediated PP2B. (A) TMSC were acutely exposed to FIR irradiation for $30 \mathrm{~min}$ in media containing $1 \mu \mathrm{M}$ Fluo-4 AM. Images of intracellular $\mathrm{Ca}^{2+}$ were visualized using a confocal microscope. Scale bar $=50 \mu \mathrm{m}$. (B) TMSC were exposed to FIR irradiation for $30 \mathrm{~min}$. PP2B protein was immunoprecipitated using an anti-PP2B antibody and PP2B activity in the precipitates was measured using a PP2B cellular activity kit. Each bar represents the mean \pm S.D. $(n=3)$. Difference was statistically significant at $* \mathrm{p}<0.05$. 


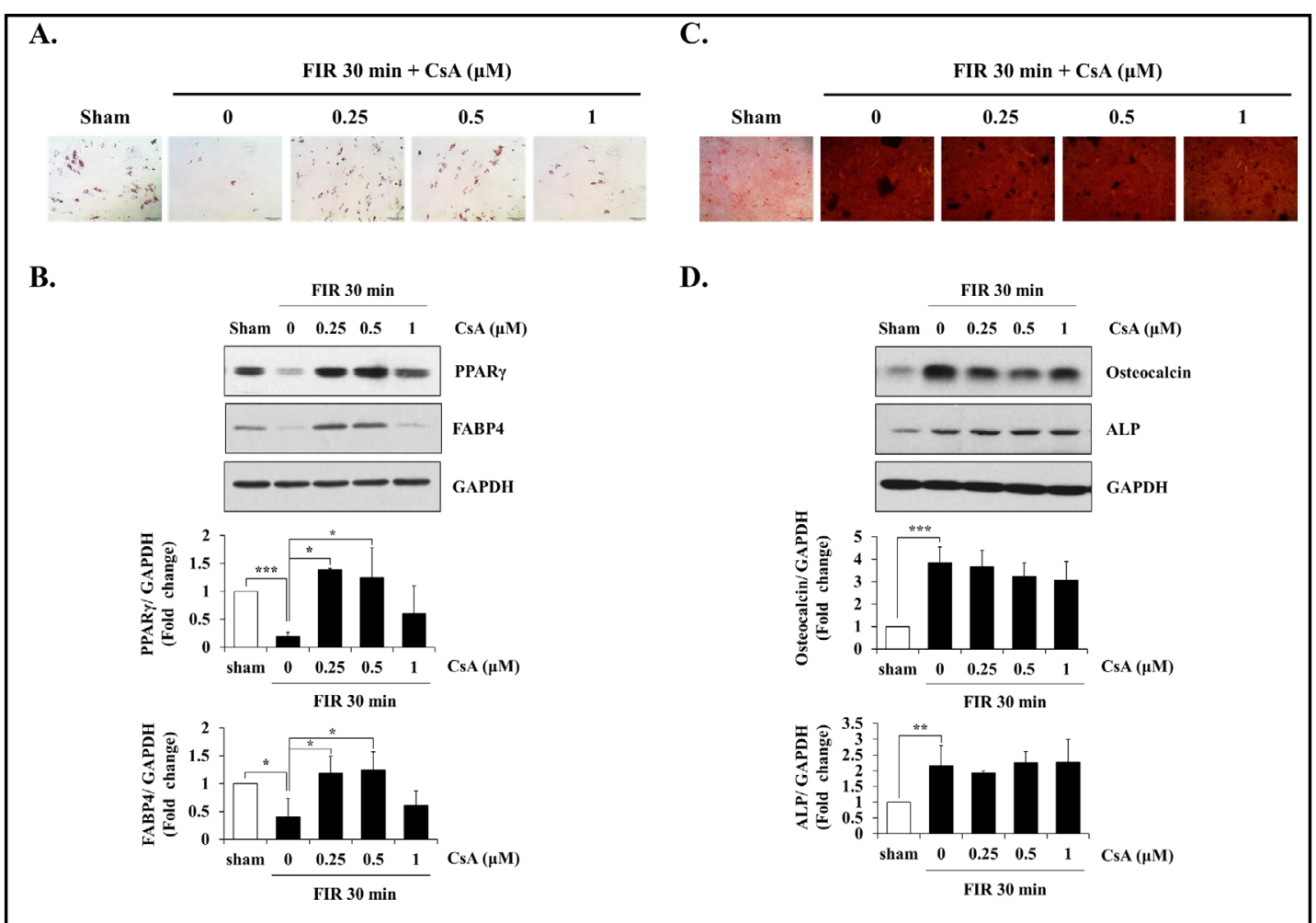

Fig. 6. Effects of activation of $\mathrm{Ca}^{2+}$-mediated PP2B on adipogenic or osteogenic differentiation of TMSC. (A-B) After pretreatment with CsA $(0,0.25,0.5$, and $1 \mu \mathrm{M})$, TMSC were exposed to FIR irradiation for 30 min and further incubated in adipogenic differentiation medium for up to 14 days. Control cells without CsA pretreatment were used as Sham. (A) Accumulation of lipid droplets and (B) protein levels of PPAR $\gamma$, FABP4, and GAPDH were measured as described in the legend of Fig. 2. (C-D) TMSC were pretreated with CsA before exposure to FIR irradiation, and further incubated in differentiation medium as described in the legend of Fig. 6A with the exception of using osteogenic medium instead of adipogenic medium. Control cells without CsA pretreatment were used as Sham. (C) Mineralization and (D) protein levels of osteocalcin, ALP, and GAPDH were measured as described in the legend of Fig. 2. The results are representative of three independent experiments, and each bar represents the mean \pm S.D. Differences were statistically significant at ${ }^{*} \mathrm{p}<0.05,{ }^{* *} \mathrm{p}<0.01$, and ${ }^{* * *} \mathrm{p}<0.001$.

been used to examine the molecular and cellular mechanisms underlying the therapeutic effects of FIR irradiation. Recent studies have identified stem cells as a potentially good source of cells for exploring mechanisms underlying the therapeutic effects of various bioactive drugs $[32,33]$. Although one recent study showed that FIR irradiation slightly alters basic characteristics of murine BM-MSC, such as proliferation and survival [23], there is no study exploring the effects of FIR irradiation on differentiation of stem cells. Here, we demonstrate that FIR irradiation of TMSC significantly inhibits adipogenesis through a mechanism mediated by a $\mathrm{Ca}^{2+}$-dependent PP2B signaling pathway. Furthermore, FIR irradiation also induces osteogenesis, but this does not appear to be related to a $\mathrm{Ca}^{2+}$-dependent PP2B signaling pathway.

One of the most important findings in this study is that FIR irradiation inhibits adipogenic differentiation and promotes osteogenic differentiation of TMSC. There are accumulated data showing that several stimuli play a dual role in regulating adipogenic and osteogenic differentiation in MSC. For example, hypoxia was reported to inhibit adipogenesis and promote osteogenesis of BM-MSC in a hypoxia-inducible factor-1-dependent manner [34], which is consistent with our current data. Furthermore, microgravity alters both adipogenesis and osteogenesis in rat BM-MSC [35], and interestingly, these effects are dose dependent; longer exposure (10 days) to microgravity inhibits adipogenesis and promotes 
osteogenesis whereas shorter exposure (3 days) shows opposite results. Similar to shorter exposure to microgravity, repeated exposure to mechanical vibration also promotes adipogenesis and inhibits osteogenesis of BM-MSC [36]. Based on our findings that a very short exposure time (only $30 \mathrm{~min}$ ) is sufficient for inhibition of adipogenesis and promotion of osteogenesis by FIR irradiation, in contrast to the relatively long exposure times required for hypoxia and microgravity to regulate the differentiation of MSC, we suggest that FIR irradiation may provide a safer and more effective therapeutic treatment.

Unlike the previous study showing that heat stimulation alone significantly reduces the early adipogenesis of 3T3-L1 preadipocytes [37], we found that heat alone did not simulate the inhibitory effect of FIR irradiation on adipogenesis of TMSC, suggesting a heatindependent mechanism of FIR irradiation. At present, the reason for these inconsistent results has not been identified, but it may be attributable to the different conditions used: we used a $38^{\circ} \mathrm{C}$ heating system and TMSC whereas the previous study used a $>43^{\circ} \mathrm{C}$ heating system and 3T3-L1 preadipocytes. Nonetheless, it is possible that the particular form of electromagnetic energy, but not the form of heat energy itself, produced by FIR plays a role in inhibiting adipogenesis of TMSC. In this regard, we also reported the heat-independent inhibitory effect of FIR irradiation on EC growth and angiogenesis [28]. However, our data showed partial heat dependence for FIR irradiation-stimulated osteogenic differentiation in TMSC, which reproduces previous data showing that heat stress at $38-40^{\circ} \mathrm{C}$ promotes osteogenesis of dental follicle stem cells [38].

Adipogenic and osteogenic differentiation of MSC are balanced by a transcriptional modulator, TAZ. TAZ is a coactivator of Runx2, which regulates osteocalcin expression to promote osteogenic differentiation, but simultaneously inhibits adipogenic differentiation by directly inhibiting PPAR $\gamma$-dependent transcriptional events essential for adipogenesis [29]. Because our data showed that FIR irradiation inhibited adipogenic differentiation and stimulated osteogenic differentiation in TMSC, we predicted that TAZ might be involved in the FIR irradiation-induced TMSC differentiation into adipocytes and osteocytes. Previous reports showed that a reduced protein level of TAZ promotes adipogenic differentiation and inhibits osteogenic differentiation of MSC [39]. Surprisingly, however, FIR irradiation clearly decreased, rather than increased, the protein expression of TAZ. Based on this finding, we suggest that there is no evidence for involvement of TAZ in the dual effects of FIR irradiation on the adipogenic and osteogenic differentiation of TMSC; however, we cannot exclude involvement of some factor(s) other than TAZ in the observed effects of FIR irradiation in TMSC.

$\mathrm{Ca}^{2+}$ is an important mediator involved in various cellular processes, such as cell growth, apoptosis, and differentiation. In particular, intracellular and extracellular $\mathrm{Ca}^{2+}$ concentrations have been reported to affect adipogenic or osteogenic differentiation [40-42]. We previously reported that FIR irradiation increased intracellular $\mathrm{Ca}^{2+}$ levels in BAEC, mediating increased eNOS phosphorylation at serine 1977 and thus increasing NO production [21]. With respect to the increased levels of intracellular $\mathrm{Ca}^{2+}$, the current data using TMSC reproduce our previous data in BAEC. Using CsA, we further demonstrate that $\mathrm{Ca}^{2+}$-dependent PP2B activity mediates the regulatory effect of FIR irradiation on adipogenesis, but not osteogenesis, of TMSC. Consistent with our data, a previous study showed that CsA attenuates adipogenesis of 3T3-L1 preadipocytes induced by the $\mathrm{Ca}^{2+}$ ionophore ionomycin [26]. Furthermore, the authors of this previous study also reported that reversal of adipogenesis by CsA is mediated by the suppression of proadipogenic transcription factors (i.e., PPAR $\gamma$ and C/EBP $\alpha$ ). Taking these findings together, it is clear that PP2B plays an important role in adipogenesis of several cells, including TMSC. Interestingly, our study showed that CsA (up to $0.5 \mu \mathrm{M}$ ) clearly reversed the inhibitory effect of FIR irradiation on adipogenesis. This reversal was also seen at a higher dose $(1 \mu \mathrm{M})$ although it was not statistically significant. At present, we cannot explain why a higher dose of CsA attenuated reversal of the inhibitory effect on adipogenesis by FIR irradiation. A previous study reported that treatment with CsA $(1 \mu \mathrm{g} / \mathrm{mL})$ alone had no effect on adipogenic differentiation of 3T3-L1 preadipocytes [26], although another study reported that a higher dose $(10 \mu \mathrm{g} / \mathrm{mL})$ in itself inhibits the adipogenic differentiation of 


\section{Cellular Physiology Cell Physiol Biochem 2019;52:240-253

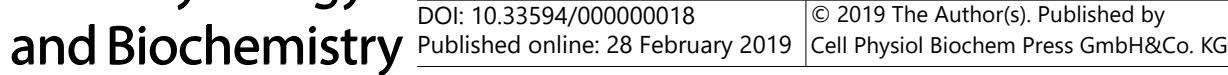 \\ Kim et al.: Far-Infrared Irradiation and Mesenchymal Stem Cell Differentiation}

3T3-L1 preadipocytes [43]. A concentration of $1 \mu \mathrm{g} / \mathrm{mL}$ CsA is equivalent to $\sim 0.8 \mu \mathrm{M}$. Based on these previous studies, it is likely that under our experimental conditions CsA was an inhibitor of PP2B but not an inhibitor of adipogenic differentiation, which further validates our conclusion that the inhibitory effect of FIR irradiation on adipogenic differentiation is mediated by PP2B activation. Regarding the involvement of PP2B in osteogenic differentiation, it was reported that deficiency in PP2B catalytic subunit A exhibited osteoporosis in vivo and in vitro [27], suggesting a role for activation of PP2B in promoting osteogenic differentiation. However, from our findings, it seems unlikely that the stimulation of osteogenesis by FIR irradiation is mediated by PP2B activation. Identifying signaling molecules other than PP2B involving in FIR irradiation-induced osteogenic differentiation of TMSC is important and needs further investigations. Previously, we reported that $\mathrm{Ca}^{2+} /$ calmodulin-dependent protein kinase II (CaMKII) plays an important role in promoting osteogenesis of human BM-MSC by the peptide with a novel collagen-binding motif derived from osteopontin [44]. Consistent with our previous study, a recent study also showed that a constant electric field induces osteogenic differentiation of rat $\mathrm{BM}-\mathrm{MSC}$ on $\mathrm{TiO}_{2}$ nanotubular layer via CaMKII [45]. With these findings, together with the previous data showing that FIR irradiation significantly activates CaMKII in BAEC [21], it seems likely that CaMKII is a potential candidate protein in stimulating osteogenic differentiation of TMSC. Further study is needed to clarify this issue.

Obesity is a medical condition of excessive fat accumulation in adipose tissues. Adipocytes, which are of mesodermal origin, are generally known to be derived from stem cells and are produced through a series of differentiation processes. Abnormal development of MSC into adipocytes leads to hyperplasia of adipocytes, which may cause obesity. Although the direct link between MSC and hyperplasia of adipocytes is not known, our study suggests that FIR irradiation can be used as a tool to understand its underlying mechanism and perhaps to treat obesity. In this regard, FIR sauna therapy was reported to significantly reduce body weight and body fat in obese patients [46]. There are also a few findings of in vivo beneficial effects of FIR irradiation on bone formation; for example, FIR irradiation was reported to promote bone-forming activity of osteoblasts and increase bone mineral density when natural FIR ceramics were implanted under the periosteum of rat skull [47]. Our current data, together with these previous findings, may extend the potential use of FIR therapy in clinical applications to treat obesity and repair bone defects.

\section{Conclusion}

We demonstrate that FIR irradiation inhibits adipogenic differentiation and promotes osteogenic differentiation of TMSC. Furthermore, the inhibition of adipogenic differentiation by FIR irradiation is mediated at least in part by $\mathrm{Ca}^{2+}$-dependent PP2B activation, but the stimulation of osteogenesis is unlikely to be PP2B-dependent. Lastly, our findings may advance our understanding of the molecular and cellular mechanism by which FIR therapy decreases body fat in obese patients and repairs bone defects.

\section{Acknowledgements}

This study was supported by the Basic Science Research Program through the National Research Foundation of Korea (NRF) funded by the Ministry of Science, ICT \& Future Planning (2017M3A9B3063636), and partly by The Korea Health Technology R\&D Project through the Korea Health Industry Development Institute (KHIDI), funded by the Ministry of Health \& Welfare, Republic of Korea (HI16C2207). 


\section{Cellular Physiology Cell Physiol Biochem 2019;52:240-253 \begin{tabular}{l|l|l}
\cline { 2 - 3 } DOl: 10.33594/000000018 & (c) 2019 The Author(s). Published by
\end{tabular} and Biochemistry Published online: 28 February 2019 Cell Physiol Biochem Press GmbH\&Co. KG \\ Kim et al.: Far-Infrared Irradiation and Mesenchymal Stem Cell Differentiation}

\section{Disclosure Statement}

All authors have declared that there is no conflict of interests.

\section{References}

1 Fortier LA: Stem cells: classifications, controversies, and clinical applications. Vet Surg 2005;34:415-423.

- 2 Nichols J, Smith A: The origin and identity of embryonic stem cells. Development 2011;138:3-8.

- 3 Lee RH, Kim B, Choi I, Kim H, Choi HS, Suh K, Bae YC, Jung JS: Characterization and expression analysis of mesenchymal stem cells from human bone marrow and adipose tissue. Cell Physiol Biochem 2004;14:311324.

4 Peister A, Mellad JA, Larson BL, Hall BM, Gibson LF, Prockop DJ: Adult stem cells from bone marrow (MSCs) isolated from different strains of inbred mice vary in surface epitopes, rates of proliferation, and differentiation potential. Blood 2004;103:1662-1668.

5 Gimble J, Guilak F: Adipose-derived adult stem cells: isolation, characterization, and differentiation potential. Cytotherapy 2003;5:362-369.

6 Lim J, Razi ZRM, Law JX, Nawi AM, Idrus R BH, Chin TG, Mustangin M, Ng MH: Mesenchymal stromal cells from the maternal segment of human umbilical cord is ideal for bone regeneration in allogenic setting. Tissue Eng Regen Med 2018;15:75-87.

7 See EY, Toh SL, Goh JC: Multilineage potential of bone-marrow-derived mesenchymal stem cell cell sheets: implications for tissue engineering. Tissue Eng Part A 2010;16:1421-1431.

8 Chamberlain G, Fox J, Ashton B, Middleton J: Concise review: Mesenchymal stem cells: their phenotype, differentiation capacity, immunological features, and potential for homing. Stem Cells 2007;25:2739-2749.

9 Ryu KH, Cho KA, Park HS, Kim JY, Woo SY, Jo I, Choi YH, Park YM, Jung SC, Chung SM, Choi BO, Kim HS: Tonsil-derived mesenchymal stromal cells: evaluation of biologic, immunologic and genetic factors for successful banking. Cytotherapy 2012;14:1193-1202.

10 Jung N, Park S, Choi Y, Park JW, Bin Hong Y, Park HHC, Yu Y, Kwak G, Kim HS, Ryu KH, Kim JK, Jo I, Choi BO, Jung SC: Tonsil-derived mesenchymal stem cells differentiate into a Schwann cell phenotype and promote peripheral nerve regeneration. Int J Mol Sci 2016;17:pii:E1867.

11 Park S, Choi Y, Jung N, Kim J, Oh S, Yu Y, Ahn J H, Jo I, Choi BO, Jung SC: Autophagy induction in the skeletal myogenic differentiation of human tonsil-derived mesenchymal stem cells. Int J Mol Med 2017;39:831-840.

12 Kim SY, Kim YR, Park WJ, Kim HS, Jung SC, Woo SY, Jo I, Ryu KH, Park JW: Characterisation of insulinproducing cells differentiated from tonsil derived mesenchymal stem cells. Differentiation 2015;90:27-39.

13 Yu Y, Lee SY, Yang EJ, Kim HY, Jo I, Shin SJ: Expression of tenocyte lineage-related factors from tonsil-derived mesenchymal stem cells. Tissue Eng Regen Med 2016;13:162-170.

14 Park YS, Kim HS, Jin YM, Yu Y, Kim HY, Park HS, Jung SC, Han KH, Park YJ, Ryu KH, Jo I: Differentiated tonsilderived mesenchymal stem cells embedded in matrigel restore parathyroid cell functions in rats with parathyroidectomy. Biomaterials 2015;65:140-152.

15 Jo YI, Kim G, Jin YM, Park YJ, Kim HS, Park YS: Intracellular remodeling and accumulation of aberrant lysosomes in differentiation of tonsil-derived mesenchymal stem cells into parathyroid-like cells. Tissue Eng Regen Med 2017;14:411-420.

16 Khatri R, Arad M, Ortlip T, Portney BA, Meltzer WA, Diaconu S, Silipino LE, Wang Y, Kaetzel DM, Taylor RJ, Zalzman M: Harvesting multipotent progenitor cells from a small sample of tonsillar biopsy for clinical applications. Stem Cell Res Ther 2017;8:174.

17 Shin SC, Seo Y, Park HY, Jung DW, Shin TH, Son H, Kim YK, Lee JC, Sung ES, Jang JY, Kim HS, Lee BJ: Regenerative potential of tonsil mesenchymal stem cells on surgical cutaneous defect. Cell Death Dis 2018;9:183.

18 Kim YH, Cho KA, Park M, Kim HS, Park JW, Woo SY, Ryu KH: Conditioned medium from tonsil-derived mesenchymal stem cells relieves CCl4-induced liver fibrosis in mice. Tissue Eng Regen Med 2018; DOI:10.1007/s13770-018-0160-8.

19 Ziegelberger G, Repacholi M: International commission on non-ionizing radiation protection. Prog Biophys Mol Bio 2006;92:1-3. 


\section{Cellular Physiology Cell Physiol Biochem 2019;52:240-253 \begin{tabular}{l|l|l}
\cline { 2 - 3 } DOI: 10.33594/000000018 & (c) 2019 The Author(s). Published by
\end{tabular} and Biochemistry Published online: 28 February 2019 Cell Physiol Biochem Press GmbH\&Co. KG

20 Beever R: Far-infrared saunas for treatment of cardiovascular risk factors: summary of published evidence. Can Fam Phys 2009;55:691-696.

21 Park JH, Lee S, Cho DH, Park YM, Kang DH, Jo I: Far-infrared radiation acutely increases nitric oxide production by increasing $\mathrm{Ca} 2+$ mobilization and $\mathrm{Ca} 2+$ /calmodulin-dependent protein kinase II-mediated phosphorylation of endothelial nitric oxide synthase at serine 1179. Biochem Biophys Res Commun 2013;436:601-606.

22 Leung TK, Chan CF, Lai PS, Yang CH, Hsu CY, Lin YS: Inhibitory effects of far-infrared irradiation generated by ceramic material on murine melanoma cell growth. Int J Photoenergy 2012; DOI:10.1155/2012/646845.

23 Jeong YM, Cheng XW, Lee S, Lee KH, Cho H, Kang JH, Kim W: Preconditioning with far-infrared irradiation enhances proliferation, cell survival, and migration of rat bone marrow-derived stem cells via CXCR4-ERK pathways. Sci Rep 2017;7:13718.

24 Matsuda S, Koyasu S: Mechanisms of action of cyclosporine. Immunopharmacology 2000;47:119-125.

25 Dumont FJ: FK506, an immunosuppressant targeting calcineurin function. Curr Med Chem 2000;7:731748.

26 Neal JW, Clipstone NA: Calcineurin mediates the calcium-dependent inhibition of adipocyte differentiation in 3T3-L1 cells. J Biol Chem 2002;277:49776-49781.

27 Sun L, Blair HC, Peng Y, Zaidi N, Adebanjo OA, Wu XB, Wu XY, Iqbal J, Epstein S, Abe E, Moonga BS, Zaidi M: Calcineurin regulates bone formation by the osteoblast. Proc Natl Acad Sci USA 2005;102:17130-17135.

28 Hwang S, Lee DH, Lee IK, Park YM, Jo I: Far-infrared radiation inhibits proliferation, migration, and angiogenesis of human umbilical vein endothelial cells by suppressing secretory clusterin levels. Cancer Lett 2014;346:74-83.

29 Hong JH, Hwang ES, McManus MT, Amsterdam A, Tian Y, Kalmukova R, Mueller E, Benjamin T, Spiegelman BM, Sharp PA, Hopkins N, Yaffe MB: TAZ, a transcriptional modulator of mesenchymal stem cell differentiation. Science 2005;309:1074-1078.

30 Sobajima M, Nozawa T, Ihori H, Shida T, Ohori T, Suzuki T, Matsuki A, Yasumura S, Inoue H: Repeated sauna therapy improves myocardial perfusion in patients with chronically occluded coronary artery-related ischemia. Int J Cardiol 2013;167:237-243.

31 Shima H, Yamamoto S, Qiu J, Shincho M, Hirota S, Yoshikawa Y, Yoshikawa R, Hashimoto-Tamaoki T: Farinfrared rays control prostate cancer cells in vitro and in vivo. Cent Eur J Biol 2010;5:178-189.

32 Tran C, Damaser MS: Stem cells as drug delivery methods: application of stem cell secretome for regeneration. Adv Drug Deliv Rev 2015;82-83:1-11.

33 Kim D, Cho GS, Han C, Park DH, Park HK, Woo DH, Kim JH: Current understanding of stem cell and secretome therapies in liver diseases. Tissue Eng Regen Med 2017;14:653-665.

34 Wagegg M, Gaber T, Lohanatha FL, Hahne M, Strehl C, Fangradt M, Tran CL, Schonbeck K, Hoff P, Ode A, Perka C, Duda GN, Buttgereit F: Hypoxia promotes osteogenesis but suppresses adipogenesis of human mesenchymal stromal cells in a hypoxia-inducible factor-1 dependent manner. PLoS One 2012;7:e46483.

35 Xue L, Li Y, Chen J: Duration of simulated microgravity affects the differentiation of mesenchymal stem cells. Mol Med Rep 2017;15:3011-3018.

36 Chen X, He F, Zhong DY, Luo ZP: Acoustic-frequency vibratory stimulation regulates the balance between osteogenesis and adipogenesis of human bone marrow-derived mesenchymal stem cells. Biomed Res Int 2015;2015:540731.

37 Ezure T, Amano S: Heat stimulation reduces early adipogenesis in 3T3-L1 preadipocytes. Endocrine 2009;35:402-408.

38 Rezai Rad M, Wise GE, Brooks H, Flanagan MB, Yao S: Activation of proliferation and differentiation of dental follicle stem cells (DFSCs) by heat stress. Cell Prolif 2013;46:58-66.

39 Guo L, Cai T, Chen K, Wang R, Wang J, Cui C, Yuan J, Zhang K, Liu Z, Deng Y, Xiao G, Wu C: Kindlin-2 regulates mesenchymal stem cell differentiation through control of YAP1/TAZ. J Cell Biol 2018;217:1431-1451.

40 Shi H, Halvorsen YD, Ellis PN, Wilkison WO, Zemel MB: Role of intracellular calcium in human adipocyte differentiation. Physiol Genomics 2000;3:75-82.

- 41 Lange-Consiglio A, Corradetti B, Meucci A, Perego R, Bizzaro D, Cremonesi F: Characteristics of equine mesenchymal stem cells derived from amnion and bone marrow: in vitro proliferative and multilineage potential assessment. Equine V J 2013;45:737-744. 


\section{Cellular Physiology and Biochemistry}

Cell Physiol Biochem 2019;52:240-253

42 Cheng S, Wang W, Lin Z, Zhou P, Zhang X, Zhang W, Chen Q, Kou D, Ying X, Shen Y, Cheng X, Yu Z, Peng L, Lu C: Effects of extracellular calcium on viability and osteogenic differentiation of bone marrow stromal cells in vitro. Hum Cell 2013;26:114-120.

43 Ho IC, Kim JH, Rooney JW, Spiegelman BM, Glimcher LH: A potential role for the nuclear factor of activated T cells family of transcriptional regulatory proteins in adipogenesis. Proc Natl Acad Sci USA 1998;95:15537-15541.

44 Sim MK, Kim MK, Bae YS, Jo I, Lee SJ, Chung CP, Park YJ, Min do S: A novel collagen binding peptide promotes osteogenic differentiation via $\mathrm{Ca} 2+$ /calmodulin-dependent protein kinase II/ERK/AP-1 signaling pathway in human bone marrow-derived mesenchymal stem cells. Cell Signal 2008;20:613-624.

45 Park J, Mazare A, Schneider H, von der Mark K, Fischer MJ, Schmuki P: Electric field-induced osteogenic differentiation on $\mathrm{TiO}_{2}$ nanotubular layer. Tissue Eng Part C 2016;22:809-821.

46 Biro S, Masuda A, Kihara T, Tei C: Clinical implications of thermal therapy in lifestyle-related diseases. Exp Biol Med 2003;228:1245-1249.

47 Dolgorsuren A, Yamashita K, Dalkhsuren SO, Sumida K, Seki S, Kitamura S: The ceramics radiating far infrared ray energy (Rhyolite) promote the formation of bone. J Hard Tissue Biol 2014;23:423-434. 